\title{
Going home after esophagectomy: The story is not over yet
}

\author{
Yaron Shargall, MD, FRCSC
}

\author{
From the Division of Thoracic Surgery, Department of Surgery, McMaster University Faculty of Health Sciences, \\ St Joseph's Healthcare, Hamilton, Ontario, Canada. \\ Disclosures: Author has nothing to disclose with regard to commercial support. \\ Received for publication Sept 23, 2018; accepted for publication Sept 24, 2018. \\ Address for reprints: Yaron Shargall, MD, FRCSC, St Joseph's Healthcare Hamilton, T2105-50 Charlton Ave E, \\ Hamilton, ON L8N 4A6, Canada (E-mail: shargal@mcmaster.ca). \\ J Thorac Cardiovasc Surg 2018;156:2338-9 \\ $0022-5223 / \$ 36.00$ \\ Copyright (c) 2018 by The American Association for Thoracic Surgery \\ https://doi.org/10.1016/j.jtcvs.2018.09.080
}

Postdischarge readmissions and emergency department (ED) visits are considered an important measure of quality of care. Although there is a general agreement that most preventable readmissions and ED visits occur within 1 month of discharge, some patient populations continue to be at risk for a longer period. Patients who undergo esophagectomy are among the most challenging populations in thoracic surgery, because they typically undergo surgery after neoadjuvant chemotherapy and radiation, and the impact of the surgical intervention itself is substantial. As such, it is not surprising that morbidity and mortality in this group of patients remain substantial. In the era of enhanced recovery after surgery pathways, many centers have been looking at pathways for an early discharge after esophagectomy, with some encouraging outcomes. $^{1}$ Those reports are mostly retrospective, however, and they typically assess early (up to 30 postoperative days) outcomes.

In this issue of the Journal, Kidane and colleagues ${ }^{2}$ report a single-center, retrospective, observational cohort study in which they assessed reasons for ED visits among patients discharged home after esophagectomy. They demonstrated that at a follow-up of 1 year, more than $30 \%$ of the patients had presented at least once to the ED, mostly for feeding tube problems (almost $40 \%$ ), followed by dysphagia or stricture and dyspnea (around $10 \%$ of the admissions each). They also found that higher income and minimally invasive surgery techniques were associated with more ED visits and that living far away from the hospital and having surgery in the later years of the observed study period were associated with a lower risk of ED visits. Intriguingly (for this specific population), feeding tube issues continued to be a dominant cause for repeated ED visits beyond the first encounter.

This is an important study, but it is not without limitations. First, Kidane and colleagues ${ }^{2}$ checked only ED visits, rather than readmissions. It is very possible that a fraction of patients (perhaps a significant fraction) were admitted to the hospital directly through postoperative follow-up clinics or were transferred directly from a different hospital to the original operating hospital. This

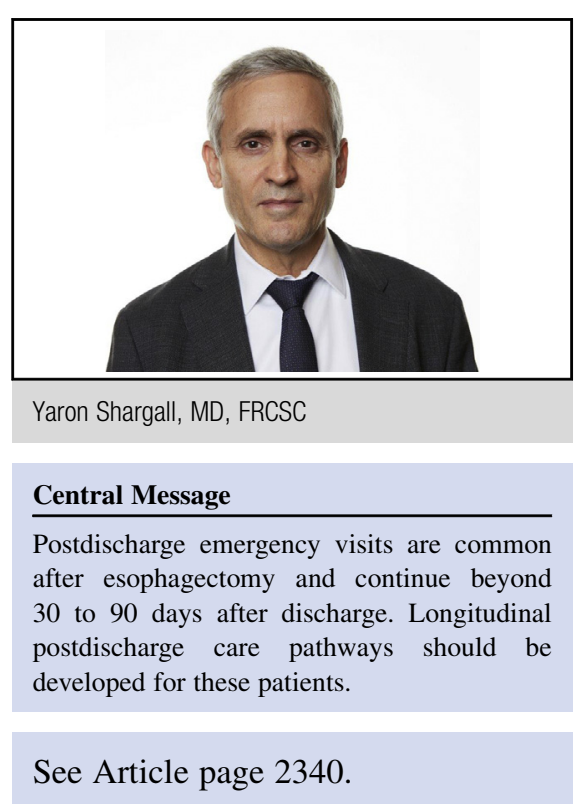

might account for a significant proportion of patients not covered by the current analysis. Secondly, the study of Kidane and colleagues ${ }^{2}$ provides data only for those patients admitted to their center, potentially missing a significant proportion of patients admitted to a different, local hospital. Indeed, it has been shown that readmissions and ED admissions to a hospital different than the original index hospital are associated with worse prognosis., Moreover, the finding that patients living far away from the operating hospital had lower risk of ED visits may be a reflection of the fact that some ED visits (to different, nonlocal, hospitals) were simply not captured. Those other, presumably uncaptured visits may have been for different etiologies, and as such their capture would have changed the analysis in a meaningful way. Finally, it is surprising that feeding tube-related problems were the leading cause for ED visits in the study of Kidane and colleagues. ${ }^{2}$ Previous analyses have shown that leading readmission and ED visit etiologies for those patients are typically related to respiratory complications, anastomotic leakages, and infections. Indeed, in a contemporary report by Bhagat and associates ${ }^{5}$ analyzing the 2012 to 2015 American College of Surgeons National Surgical Quality Improvement Program database for postoperative unplanned readmissions after esophagectomy, the main reasons for readmissions were equally divided between pulmonary, infectious and gastrointestinal 
(25\% each) and that more that $5 \%$ were related to venous thromboembolic events. Gastrostomy- or jejunostomyrelated problems accounted for less than $1 \%$ of readmissions. As such, it is plausible to assume that patients seen in ED after esophagectomy in the study of Kidane and colleagues $^{2}$ may represent a somewhat different population, at least partially as a result of not including those patients who are readmitted through different routes.

Nevertheless, Kidane and colleagues ${ }^{2}$ do provide us with a novel and important information related to long-term ED use. They followed their patients for more than 1 year after surgery and clearly demonstrated that those patients continue to consume health care resources long after the index surgery. For those patients, the story is far from over after a successful resection and even a flawless hospital stay. Moreover, the fact that their report is an institutional one allows a significantly higher granularity of data collection and interpretation, a common problem in retrospective studies based on regional or national databases. As such, the longitudinal outcome information provided in this article is practical and beneficial for all of us who operate on those patients.

Ultimately, their work, as well as previous reports, all point to the same conclusions. (1) Patients who have undergone esophagectomy require significant amounts of attention and care even after a successful operation, uneventful hospital stay, and a seamless discharge from the hospital. In addition, they continue to require health care resources far beyond the 30- to 60-day window typically used for analysis. (2) The use of hospital stay parameters, or even 90 days after discharge, as the only surrogate for quality of care is simply not enough, as clearly demonstrated by Kidane and colleagues. ${ }^{2}$ (3) Finally, it is for us, their surgeons, to be responsible to introduce longitudinal postdischarge care pathways for those patients ${ }^{6}$ as their journey continues long after a successful post operative home discharge.

\section{References}

1. Findlay JM, Gillies RS, Millo J, Sgromo B, Marshall RE, Maynard ND. Enhanced recovery for esophagectomy: a systematic review and evidence-based guidelines. Ann Surg. 2014;259:413-31.

2. Kidane B, Kaaki S, Hirpara DH, Shen YC, Bassili A, Allison F, et al. Emergency department use is high after esophagectomy and feeding tube problems are the biggest culprit. J Thorac Cardiovasc Surg. 2018;156:2340-8.

3. Staples JA, Thiruchelvam D, Redelmeier DA. Site of hospital readmission and mortality: a population-based retrospective cohort study. CMAJ Open. 2014;2: E77-85.

4. Zafar SN, Shah AA, Channa H, Raoof M, Wilson L, Wasif N. comparison of rates and outcomes of readmission to index vs nonindex hospitals after major cancer surgery. JAMA Surg. 2018;153:719-27.

5. Bhagat R, Bronsert MR, Juarez-Colunga E, Weyant MJ, Mitchell JD, Glebova WG, et al. Postoperative complications drive unplanned readmissions after esophagectomy for cancer. Ann Thorac Surg. 2018;105:1476-82.

6. Shargall Y, Hanna WC, Schneider L, Schieman C, Finley CJ, Tran A, et al. The integrated comprehensive care program: a novel home care initiative after major thoracic surgery. Semin Thorac Cardiovasc Surg. 2016;28:574-82. 